\title{
Upconversion Enhancement via Functionalization of Plasmonic Nanoparticles
}

\author{
N Barker ${ }^{1}$, E Westbrook ${ }^{2}$, J Krause ${ }^{2}$, P Zhang $^{2}$ \\ ${ }^{1}$ University of Cincinnati, Cincinnati, $\mathrm{OH},{ }^{2}$ University of Cincinnati Department of Chemistry, \\ Cincinnati, $\mathrm{OH}$ \\ natebarker52@gmail.com
}

Recently, many techniques have been explored to increase the efficiency of triplet-triplet annihilation upconversion (TTAUC) materials by locking the sensitizers and acceptors into fixed locations. Previous work has shown that isolating the position of the sensitizer onto a gold nanoparticle can increase the efficiency of the TTAUC process. This increase is believed to derive from the plasmonic structure of the nanoparticles. Our current direction is to isolate the acceptor on a gold nanoparticle. The location of the acceptor can be tuned and will afford an increase in the efficiency of the TTAUC system. Ultimately, the goal is to increase the efficient harnessing of energy from our sun by utilizing the plasmonic nature of gold nanoparticles. Crystallography is at the heart of the characterization of these novel acceptor compounds.

Acta Cryst. (2020). A76, a176 